\title{
Environmental DNA survey of the Winter Salmonosphere in the Gulf of Alaska
}

\author{
Christoph M. Deeg ${ }^{1}$, Svetlana Esenkulova ${ }^{2}$, Shaorong Li ${ }^{3}$, Brian P.V. Hunt ${ }^{4,5,6}$, Ben J.G. Sutherland ${ }^{3}$, Angela \\ Schulze $^{3}$, and Kristina M. Miller ${ }^{1,3}$ \\ ${ }^{1}$ Forest and Conservation Sciences, University of British Columbia, Vancouver, BC, Canada \\ ${ }^{2}$ Pacific Salmon Foundation, Vancouver, BC, Canada \\ ${ }^{3}$ Fisheries and Oceans Canada, Pacific Biological Station, Nanaimo, BC, Canada \\ ${ }^{4}$ Department of Earth, Ocean and Atmospheric Sciences, University of British Columbia, 2039-2207 Main Mall, \\ Vancouver, BC V6T 1Z4, Canada \\ ${ }^{5}$ Institute for the Oceans and Fisheries, University of British Columbia, 2202 Main Mall, Vancouver, BC V6T 1Z4, \\ Canada \\ ${ }^{6}$ Hakai Institute, PO Box 309, Heriot Bay, BC VOP 1HO, Canada
}

Keywords: environmental DNA, Gulf of Alaska, salmon, Northeast Pacific, winter

Environmental DNA (eDNA) is an emerging field to study the diversity and distribution of aquatic communities without the need to capture individuals. It utilizes sequencing of traces of DNA left behind in the environment by organisms to provide an unbiased account of species composition and distribution in an environment without the need for invasive sampling methods (Rees et al. 2014). The International Year of the Salmon (IYS) Gulf of Alaska (GoA) expeditions were launched in 2019 and 2020 to illuminate the factors influencing the survival of Pacific salmon in the open ocean. The winter months, when open-ocean conditions might critically impact ocean survival of first ocean-winter juvenile and subadult salmon, are the least understood but could largely determine stock performance (Beamish and Mahnken 2001; Naydenko et al. 2016; Shuntov et al. 2017; Nagasawa 2000; Ishida et al. 2000). Despite progress on salmon marine ecology during the winter, questions regarding the health and survival of salmon during this period remain unanswered, particularly in the open ocean. Specifically, the impact of predators and competitors has been notoriously difficult to assess due to the scarcity of data, in part due to the difficulty of catching large highly mobile predators of salmon in trawl surveys. Accordingly, we collected water samples during the GoA expeditions and performed an eDNA survey on the samples with the goal of describing the salmonosphere (i.e., the relative abundance and distribution of Pacific salmon, as well as their prey, competitors, and predators).

Water samples of $5 \mathrm{~L}$ were collected at 2-4 m below surface using a Niskin bottle at all oceanographic stations during the 2019 and 2020 GoA expeditions. Duplicate $2 \mathrm{~L}$ subsamples were filtered into $0.22 \mu \mathrm{m}$ Sterivex cartridge filters and preserved at $-80^{\circ} \mathrm{C}$ for transport back to the laboratory. All sampling equipment was cleaned with $1.6 \%$ sodium hypochlorite solution followed by rinsing with distilled water. In 2020, an additional step of $10 \%$ sodium thiosulfate rinse was added to neutralize any residual bleach from the system. Upon return from the field, DNA was extracted from the filter cartridges using the DNeasy extraction kit (Qiagen). To assess chordate and cephalopod species diversity we amplified conserved regions of mitochondrial $16 \mathrm{~S}$ gene using primers designed by Deagle et al. (2009). To speciate salmonids we similarly targeted the cytochrome oxidase I (COI) gene of Pacific salmon (Thomas et al. 2017). The $16 \mathrm{~S}$ and COI amplifications were normalized prior to library preparation using a normalization plate to standardize the input material and retrieve similar sequence levels across the different samples. Library preparation was performed using the KAPA Low Throughput Library Preparation kit for Illumina platforms, as per manufacturer's instructions. The $16 \mathrm{~S}$ and COI samples were pooled into nine libraries sequenced in single-end $300 \mathrm{bp}$ on an Illumina MiSeq platform, with 7-18 barcoded libraries per run. Sequencing data were quality filtered, demultiplexed, and rarefied using the OBItools suite (https://git.metabarcoding.org/obitools/obitools/wikis/home). Next, unique reads were queried against the nr BLAST database using a standalone local BLAST algorithm (https://blast.ncbi.nlm.nih.gov/Blast.cgi) and results were assigned to the lowest taxonomic level using MEGAN (https://bio.tools/megan). A detailed description of the workflow can be found at https://github.com/bensutherland/eDNA_metabarcoding/. R was used for filtering and assigning species detections to samples and for statistical analysis and visualization of the data. Reads belonging to common contaminants (such as humans, pigs, chickens, and cows) were excluded from the analysis.

All correspondence should be addressed to C. Deeg. 

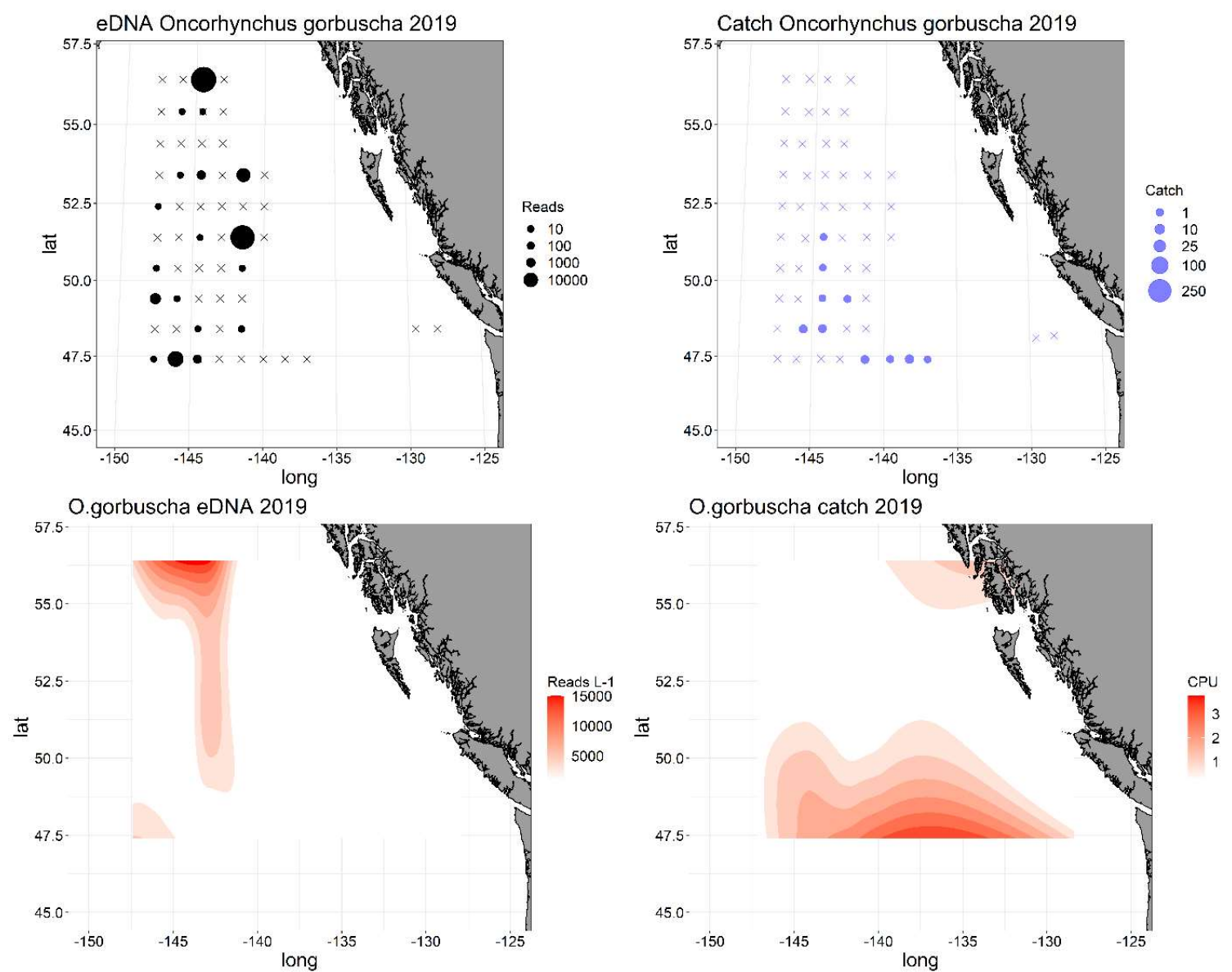

Fig. 1. Capture and eDNA detection location of pink salmon (Oncorhynchus gorbuscha) in the GoA in 2019 and the distribution density calculation based on the respective data.

eDNA was able to detect all salmon species caught in 2019 except for Chinook, which had only three individuals captured. Salmon distributions detected by eDNA were in general similar to trawl catches, with the exception of pink salmon that showed a distinct distribution center in the northwest of the survey region where no pink salmon were caught in trawls, suggesting that trawl sampling might have missed a secondary distribution center (Fig. 1).
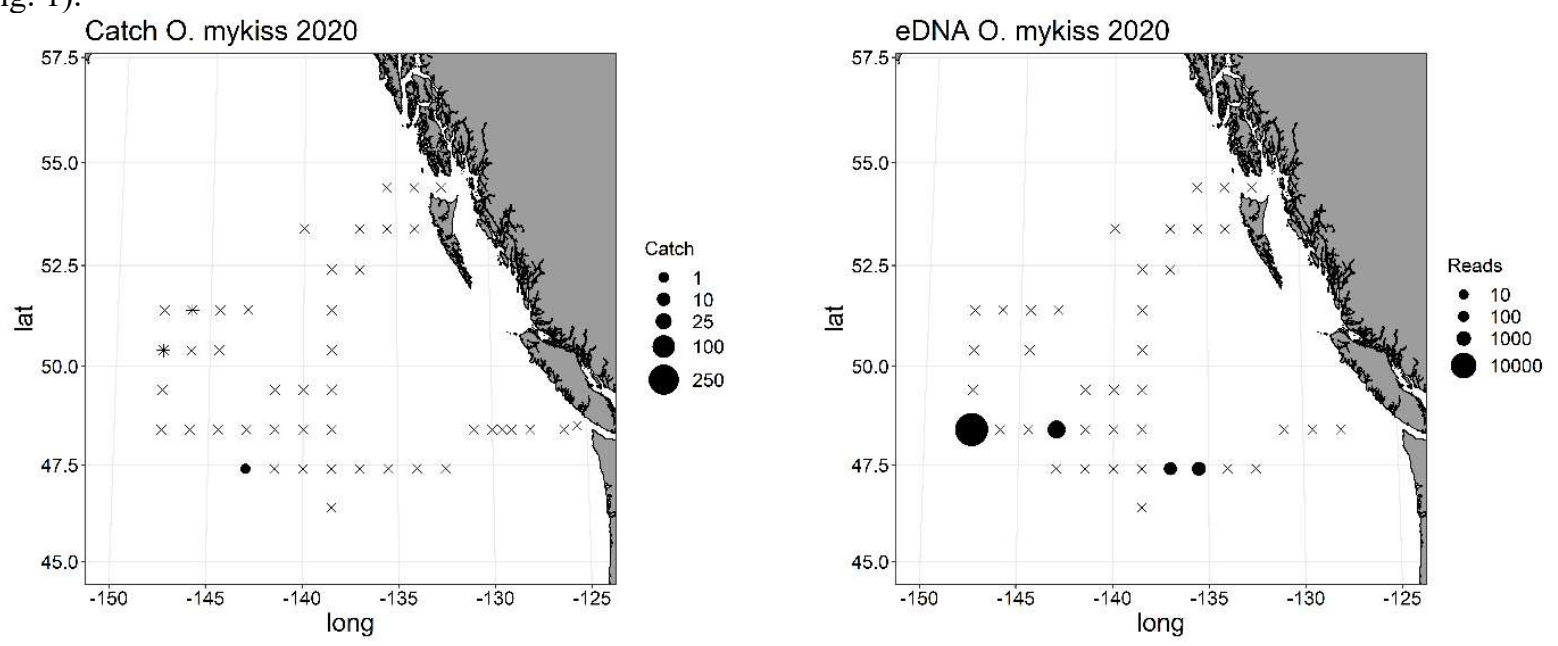

Fig. 2. Capture and eDNA detection location of steelhead (Oncorhynchus mykiss) in the GoA in 2019. 

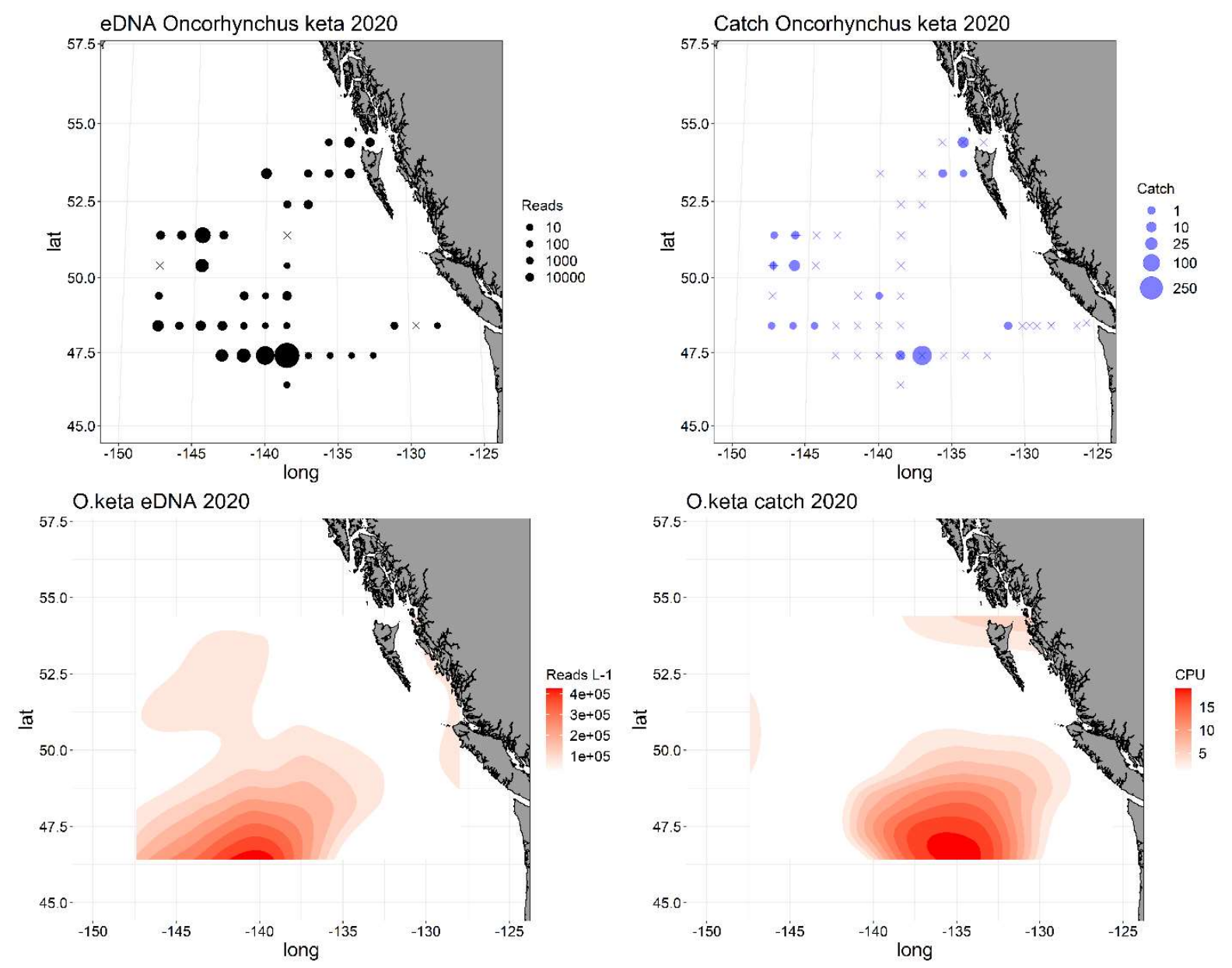

Fig. 3. Capture and eDNA detection location of chum salmon (Oncorhynchus keta) in the GoA in 2020 and the distribution density calculation based on the respective data.

In 2020, all salmon species captured by trawl were detected by eDNA and eDNA detections outnumbered trawl catches, presumably due to the increased detection sensitivity due to the addition of sodium thiosulfate to the sampling procedure. For instance, only one steelhead (Oncorhynchus mykiss) was captured in 2020, but steelhead DNA was detected at four stations during the survey (Fig. 2). The center of distribution for coho, sockeye, and pink salmon in the GoA in 2020 based on eDNA data was shifted to the west compared to trawl catches (Fig. 3). Given the half life of DNA in sea water, specifically in the winter environment, this could suggest eastward movement of these species towards the continental shelf in the study area during early spring of 2020. One main area of interest in eDNA data was the distribution and abundance of predators of salmon, as predators are notoriously difficult to assess in trawl surveys due to their low abundance and high motility. Only two potential predator species of salmon, North Pacific daggertooth (Anotopterus nikparini) and Pacific spiny dogfish (Squalus suckleyi), had been caught during the GoA expeditions. Indeed, these two species were also detected in the eDNA data. Additionally, salmon sharks (Lamna ditropis), presumed to be major predators of salmon in the open ocean, were detected during both years of the survey (Fig. 4) (Nagasawa 1998; Seitz et al. 2019). Other detections of potential predators of juvenile salmon were Dall's porpoise (Phocoenoides dalli) as well as a number of predators of presumed lesser importance such as Orca (Orcinus orca), lancetfish (Alepisaurus ferox), and Steller sea lion (Eumetopias jubatus). Dall's porpoise and salmon shark showed distribution patterns that aligned with the overall distribution of Pacific salmon in both years, suggesting that they might seek out high abundances of their prey species (Fig. 4). Specifically in 2020, where the majority of salmon appeared to be just west of the survey area, predator detections were most common on the westernmost stations (Fig. 4). Surprisingly, harbour seal (Phoca vitulina) DNA was also detected in the GoA, specifically in 2019, and seemed to show strong patterns of co-occurrence with salmon in their distribution. While this species is commonly thought to be restrained to the continental shelf, a visual observation from a crew member of the 2019 expeditions seems to confirm these unexpected detections (Brown and Mate 1983). 
Many prey and competitor species of salmon in the open ocean, such as myctophids and cephalopods, perform diurnal vertical migrations which make an adequate assessment of their abundance and distribution by conventional surface trawl surveys challenging as they spend the daytime in deep waters. As DNA remains in the environment even after a species leaves, eDNA detections of vertically migrating species were only slightly affected by time of day in contrast to trawl catches that occurred almost exclusively at night (Table 1). For instance, the distribution of abundant species like Boreopacific armhook squid (Gonatopsis borealis) and California headlightfish (Diaphus theta) showed a much more even distribution in the eDNA data compared to trawl catch data (Fig. 5). The current dataset did also allow some insights into some species of salmon prey, specifically copepods. Mesocallanus tenuicornis and Metridia pacifica were the most abundant copepod species detected in 2019 and 2020, with Mesocallanus tenuicornis dominating in the south and Metridia pacifica being dominant in the north of the GoA.
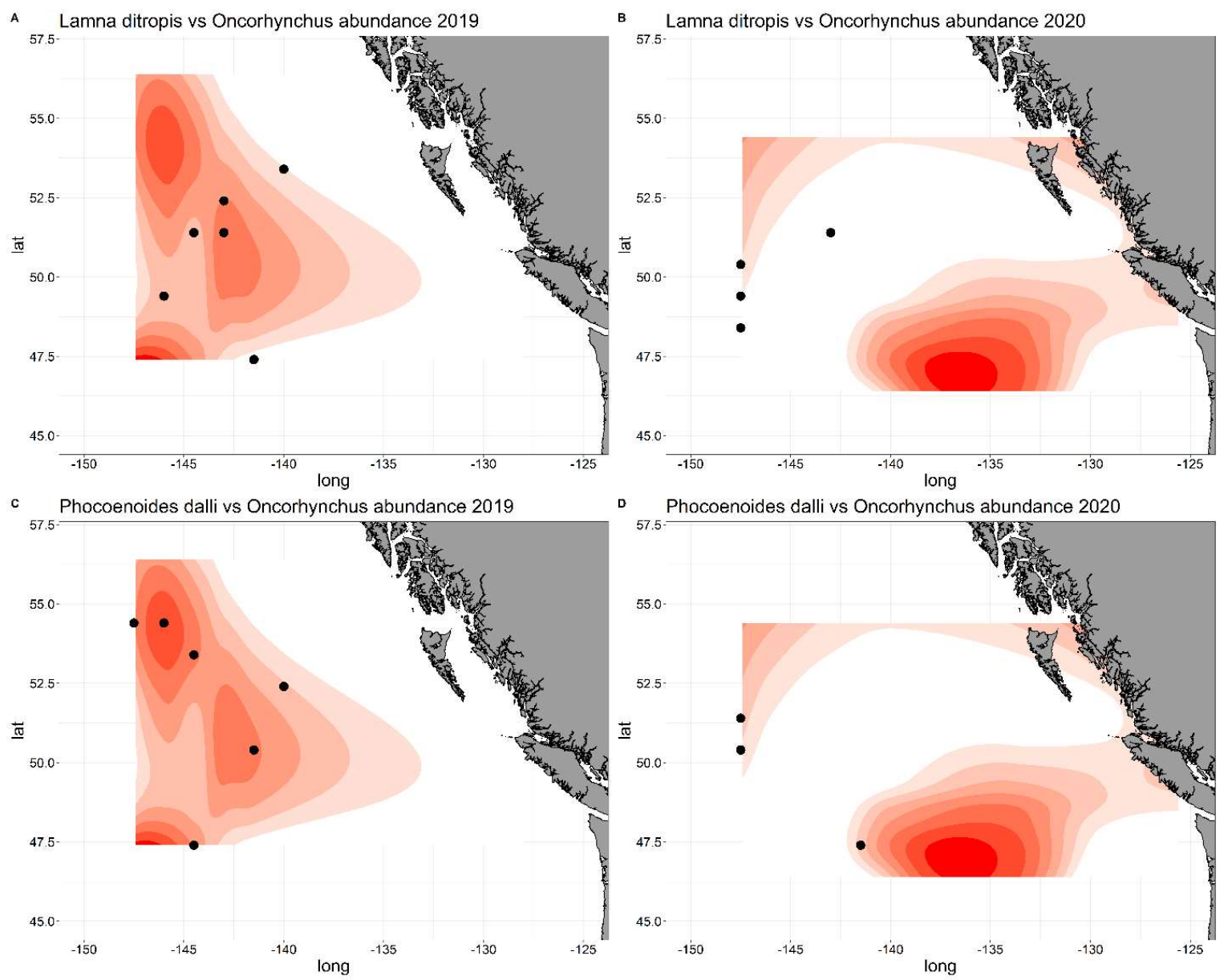

Fig. 4. eDNA detection location of salmon shark (Lamna ditropis) and Dall's porpoise (Phocoenoides dalli) in the GoA in 2019 and 2020 overlayed the distribution density all salmon species (Oncorhynchus spp.) combined.

Table 1. Proportions of detections of diurnal vertically migrating prey and competitor groups of salmon.

\begin{tabular}{c|c|c|c}
\hline Species & Time & eDNA & Trawl \\
\hline \multirow{2}{*}{ Squid } & Day & $52.7 \%$ & $20 \%$ \\
\cline { 2 - 4 } & Night & $43.7 \%$ & $91 \%$ \\
\hline \multirow{2}{*}{ Myctophids } & Day & $79.3 \%$ & $8.6 \%$ \\
\cline { 2 - 4 } & Night & $80 \%$ & $86 \%$ \\
\hline
\end{tabular}



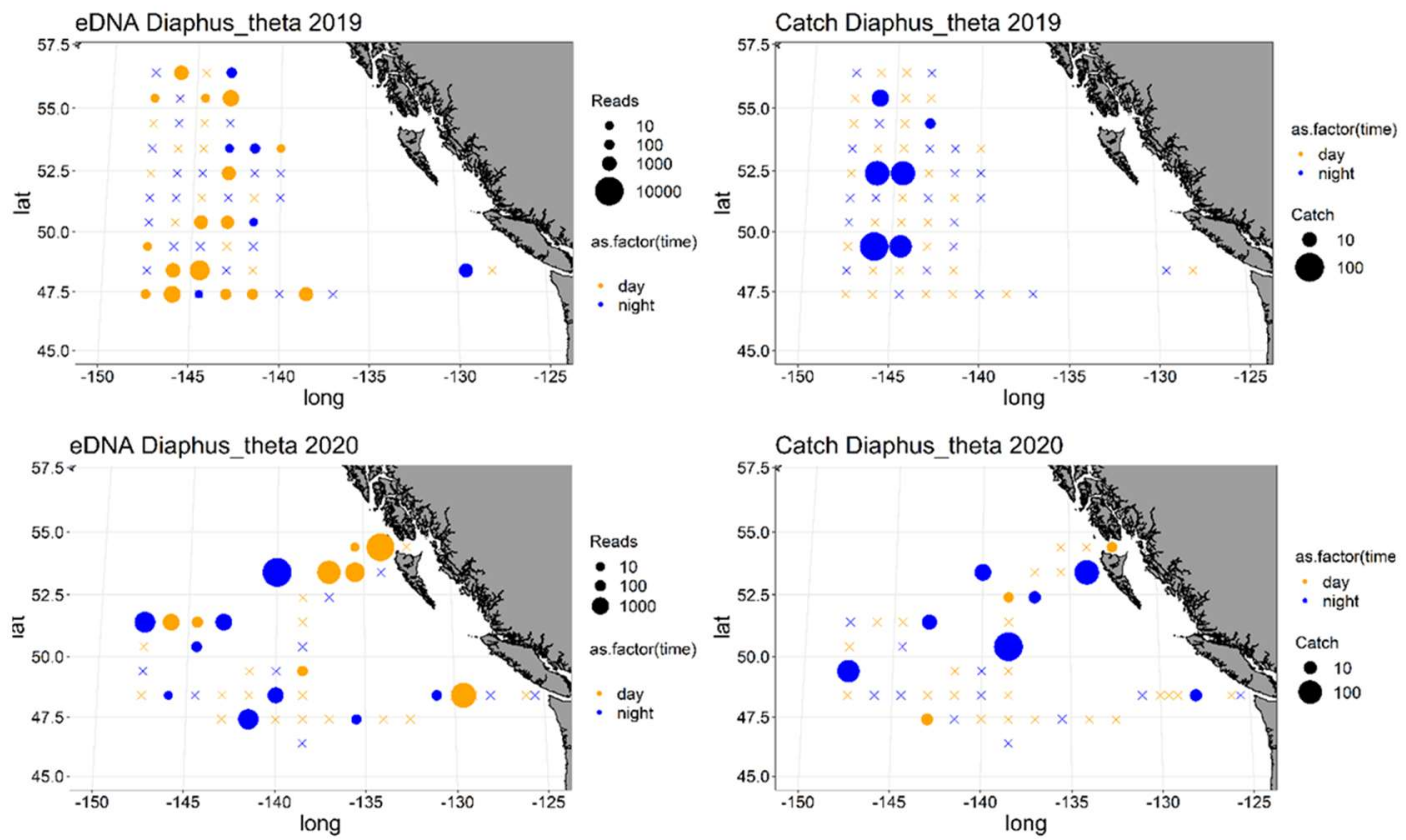

Fig. 5. Capture and eDNA detection location of California headlightfish (Diaphus theta) in the GoA in 2019 and 2020 with the time sampling time indicated by color.

Together, eDNA surveys in combination with conventional trawl surveys allow for a more holistic impression of the open ocean environment in the GoA that salmon face during the ocean winter. Specifically, interactions with predators and competitors are becoming increasingly resolved — an unprecedented resolution due to the non-invasive and unbiased nature of eDNA sampling.

\section{REFERENCES}

Beamish, R.J., and C. Mahnken. 2001. A critical size and period hypothesis to explain natural regulation of salmon abundance and the linkage to climate and climate change. Prog. Oceanogr. 49(1): 423-37.

Brown, R.F., and B.R. Mate. 1983. Abundance, movements, and feeding habits of harbor seals, Phoca vitulina, at Netarts and Tillamook Bays, Oregon. Fish. Bull. 81(2): 291-301.

Deagle, B.E., R. Kirkwood, and S.N. Jarman. 2009. Analysis of Australian fur seal diet by pyrosequencing prey DNA in faeces. Mol. Ecol. 18 (9): 2022-2038.

Ishida, Y., Y. Ueno, K. Nagasawa, and A. Shiomoto. 2000. Review of ocean salmon research by Japan from 1991 to 1998. N. Pac. Anadr. Fish Comm. Bull. 2: 191-201. (Available at https://npafc.org)

Nagasawa, K. 1998. Predation by salmon sharks (Lamna Ditropis) on Pacific salmon (Oncorhynchus Spp.) in the North Pacific Ocean. N. Pac. Anadr. Fish Comm. Bull. 1: 419-433. (Available at https://npafc.org)

Nagasawa, K. 2000. Winter zooplankton biomass in the Subarctic North Pacific, with discussion on the overwintering survival strategy of Pacific Salmon (Oncorhynchus Spp.). N. Pac. Anadr. Fish Comm. Bull 2: 21-32. (Available at https://npafc.org)

Naydenko, S.V., O.S. Temnykh, and A.L. Figurkin. 2016. Is winter the critical period in the marine life history of Pacific Salmon? N. Pac. Anadr. Fish Comm. Bull. 6: 139-52. (Available at https://npafc.org)

Rees, H.C., B.C. Maddison, D.J. Middleditch, J.R.M. Patmore, and K.C. Gough. 2014. REVIEW: The Detection of aquatic animal species using environmental DNA - a Review of eDNA as a survey tool in ecology. J. Appl. Ecol. 51(5): 1450-1459.

Seitz, A.C., M.B. Courtney, M.D. Evans, and K. Manishin. 2019. Pop-up satellite archival tags reveal evidence of intense predation on large immature Chinook salmon (Oncorhynchus tshawytscha) in the North Pacific Ocean. Can. J. Fish. Aquat. Sci. 76(9): 1608-1615.

Shuntov, V.P., O.S. Temnykh, and O.A. Ivanov. 2017. On the persistence of stereotypes concerning the marine ecology of Pacific salmon (Oncorhynchus Spp.). Russ. J. Mar. Biol. 43(7): 507-34.

Thomas, A.C., B.W. Nelson, M.M. Lance, B.E. Deagle, and A.W. Trites. 2017. Harbour seals target juvenile salmon of conservation concern. Can. J. Fish. Aquat. Sci. 74(6): 907-21. 